FIAN/TD/00-01

\title{
Once again on the equivalence theorem.
}

\author{
I.V.Tyutin * \\ I.E.Tamm Department of Theoretical Physics, \\ P.N.Lebedev Physical Institute, \\ 117924, Leninsky Prospect 53, Moscow, Russia.
}

\begin{abstract}
We present the proof of the equivalence theorem in quantum field theory which is based on a formulation of this problem in the field-anitfield formalism. As an example, we consider a model in which a different choices of natural finite counterterms is possible, leading to physically non-equivalent quantum theories while the equivalent theorem remains valid.
\end{abstract}

*E-mail: tyutin@td.lpi.ac.ru 


\section{Introduction}

The equivalence theorem in the Lagrangian quantum field theory (that states the independence of physical observables, in particular, the $S$-matrix, in quantum theory on changes of variables in the classical Lagrangian, i.e. on the choice of parametrization of the classical action) has a long story [1] - [11]. The first rigorous result is due to Borchers [2] who proves that the $S$-matrix for the field that is the local normally ordered polinomial of a free field and has non-zero in-limit coincides with the $S$-matrix for the free field, i.e. is equal to unity (on the generalization of Borchers's results for theories with interaction in the framework of the axiomatic approach see [11]). For the theories with non-zero interaction the rigorous perturbative proof of the equivalence was given in [8], [9]. In these papers, the quantum action principle was used in the form that coincides with the formal expression following, for example, from formal manipulations with the functional integral representation for the Green functions. However, in the general case, the form of quantum action principle differs from the formal expression by the so called local insertions (see sect. 3). In this paper, we present the perturbative proof of the equivalence theorem that is valid for any quantum theory renormalized with the use of the Bogoliubov $R$-operation [12] (see also [13] and reference therein). The changes of variables in classical action are treated as specific symmetries of this action. The problem of the proof of the equivalence theorem reduces to the problem of the possibility to conserve this symmetry in the quantum theory. To solve this problem, we use the generalization of the field-antifield formalism [14 to the case of global symmetries [15] - 17] and the cogomological method for studying the symmetry structure in the renormalized theory, this method was successfully applied to gauge theories

(see [18], [19] and reference therein). We show that the equivalence theorem is valid in the sense that we can always choose the finite quantum corrections (dependent on the action parametrization) to the classical action such that the physical observables and $S$-matrix do not depend on the choice of the classical action parametrization. There is a rather large arbitrariness in the choice of the finite counterterms such that we can obtain the physically nonequivalent families of quantum theories, the equivalence theorem being valid inside each of these. The paper is organized as follows. In Section 2 , we present the formal considerations of the equivalence theorem. In Section 3, we derive the basic equation for the vertex function generating functional, from which the equivalence theorem follows. In Section 4 we consider the example of the theory where the different natural choice of counterterms is possible that leads to the physically nonequivalent theories without breaking the equivalence theorem.

\section{Formal consideration}

In this section, we briefly recall the must convenient for our purposes scheme of proving the equivalence theorem. Let

$$
S_{0}=S_{0}(\varphi)=\int d x L\left(\varphi^{i}(x), \partial_{\mu} \varphi^{i}(x), \ldots\right)
$$


be a classical action. For simplicity, we assume that all the fields (which we also call variables) $\varphi^{i}(x) \equiv \varphi^{A}$ are the Bose ones,and the Lagrangian density $L$ depends on a finite number of the derivatives of the fields $\varphi^{i}(x)$ (at least, perturbatively). We consider the family of classical actions $S(\alpha, \varphi)$ :

$$
S(\alpha, \varphi)=S_{0}(\Phi(\alpha, \varphi))
$$

where the change of variables $\Phi^{A}(\alpha, \varphi)=\Phi^{i}(\alpha, \varphi ; x)=\Phi^{i}\left(\alpha, \varphi^{j}(x), \partial_{\mu} \varphi^{j}(x), \ldots\right)=$ $\varphi^{A}+O(g)$ ( $g$ denotes the total set of the coupling constants of the theory), $\Phi^{i}(0, \varphi ; x)=$ $\varphi^{i}(x)$, and its inverse are local (at least, perturbatively); the quantities

$$
f^{A}=f^{A}(\alpha, \varphi)=\frac{\partial \Phi^{B}(\alpha, \varphi)}{\partial \alpha} \frac{\delta \varphi^{A}(\alpha, \Phi)}{\delta \Phi^{B}}, \quad \varphi^{A}(\alpha, \Phi(\alpha, \varphi))=\varphi^{A}
$$

being (perturbatively) local functions of $\varphi^{i}(x)$. We obviously have

$$
S(0, \varphi)=S_{0}(\varphi)
$$

The fact that the action $S(\alpha, \varphi)$ is obtained from the action $S_{0}(\varphi)$ by the change of variables leads to the (symmetry) equation for $S(\alpha, \varphi)$ :

$$
\frac{\partial S(\alpha, \varphi)}{\partial \alpha}-f^{A}(\alpha, \varphi) \frac{\delta S(\alpha, \varphi)}{\delta \varphi^{A}}=0 .
$$

Eq. (2) is satisfied because the change of variables obeys this equation:

$$
\frac{\partial \Phi^{A}(\alpha, \varphi)}{\partial \alpha}-f^{B}(\alpha, \varphi) \frac{\delta \Phi^{A}(\alpha, \varphi)}{\delta \varphi^{B}}=0
$$

Classical theories related by a change of variables are equivalent. Naively, the same situation takes places in quantum field theory. Consider the family of the Green function generating functionals $Z(\alpha, J)$ :

$$
\begin{gathered}
Z(\alpha, J)=e^{i W(\alpha, J)}=\int D \varphi \Delta(\alpha, \varphi) e^{i\left(S(\alpha, \varphi)+J_{A} \varphi^{A}\right)}, \\
\Delta(\alpha, \varphi)=\operatorname{Det} \frac{\delta \Phi^{A}(\alpha, \varphi)}{\delta \varphi^{B}} .
\end{gathered}
$$

Evaluating the mean of symmetry equation (2) and formally integrating by parts, we obtain the equation for the Green function generating functionals:

$$
\begin{gathered}
\frac{\partial}{\partial \alpha} W(\alpha, J)+J^{A}\left\langle f^{A}\right\rangle(\alpha, J)=0 \\
\left\langle f^{A}\right\rangle(\alpha, J) \equiv \frac{1}{Z(\alpha, J)} \int D \varphi f^{A}(\alpha, \varphi) \Delta(\alpha, \varphi) e^{i\left(S(\alpha, \varphi)+J_{A} \varphi^{A}\right)},
\end{gathered}
$$

or, equivalently, the equation for the vertex function generating functional:

$$
\frac{\partial \Gamma(\alpha, \varphi)}{\partial \alpha}-\left\langle f^{A}\right\rangle(\alpha, J(\alpha, \varphi)) \frac{\delta \Gamma(\alpha, \varphi)}{\delta \varphi^{A}}=0,
$$




$$
\Gamma(\alpha, \varphi)=W(\alpha, J)-J_{A} \varphi^{A}, \quad \varphi^{A}=\frac{\delta W(\alpha, J)}{\delta J_{A}},
$$

$J$ in the functional $\left\langle f^{A}\right\rangle$ of equation (đ) being expressed in terms $\varphi$. An equation of the type (4) for the vertex function generating functional will be called the basic equation.

We assume that one-particle irreducible components of skeleton diagrams (i.e. oneparticle irreducible skeleton subdiagrams that are not contained in any other oneparticular irreducible skeleton subdiagrams) have no one-particle pole singularities with respect to momentum conjugated to the coordinates of the vertex $f^{i}(\alpha, \varphi ; x)$ (this assumption is certainly valid if all the fields are massive). Then it follows from eqs (3) or (田) (see, for example [7]), that the masses of particles and the $S$-matrix elements do not depend on $\alpha$.

The deficiency of this consideration is that none of used quantities of quantum field theory $\left(Z, W, \Gamma,\left\langle f^{A}\right\rangle\right)$ does not exist because of the known ultraviolet divergencies. We can however make a useful conclusion from this formal consideration. Really, the equivalences theorem is based on the equation of the type (3) or (国). If we establish that finite (renormalized) generating functionals satisfy the equations of the type (3) or (4), where $\left\langle f^{A}\right\rangle$ is the mean of local operator, this will imply... that masses and $S$-matrix elements do not depend on $\alpha$, which we interpret as the equivalence theorem. In the next section, we show that in any theory that can be made finite by a renormalization of the Bogoliubov $R$-operation type, we can succeed for the vertex function generating functional to satisfy the basic equation of the type (身).

\section{Basic equation}

As we said above, the fact that the action $S(\alpha, \varphi)$ is obtained from the action $S_{0}(\varphi)$ by the change of variables can be treated as the presence of the global symmetry of the action $S(\alpha, \varphi)$, whose infinitesimal form is

$$
\delta \varphi^{A}=-f^{A} \theta, \quad \delta \alpha=\theta,
$$

$\theta$ is a parameter of the global symmetry transformation. To study global symmetries in quantum field theory, it is convenient to use the field-antifield formalism [15] - [17] developed by Batalin and Vilkovisky for local (gauge) symmetries [14. We shall follow this strategy.

In accordance with the presence of the global symmetry, we introduce an additional global ghost variable $c, \varepsilon(c)=1, c^{2}=0$, and the antivariables $\varphi_{i}^{*}(x)$ with the opposite Grassman parity associated with variables $\varphi^{i}(x)$ (we do not need antivariables $\alpha^{*}, c^{*}$ ). We assign a ghost number $g h$ to every variables:

$$
g h\left(\varphi^{A}\right)=g h(\alpha)=0, \quad g h\left(\varphi_{A}^{*}\right)=-1, \quad g h()=1 .
$$

In what follows, the total set of variables is denoted by $\eta: \eta=\left\{\varphi^{A}, \varphi_{A}^{*}, \alpha, c\right\}$, the set of variables $\varphi^{A}, \alpha$ is denoted by $\xi: \xi=\left\{\varphi^{A}, \alpha\right\}$, the dependence on these variables being explicitly indicated.

We take the master action $\mathcal{S}(\eta), \varepsilon(\mathcal{S})=g h(S)=0$, to be:

$$
\mathcal{S}(\eta)=S(\xi)-\varphi_{A}^{*} f^{A}(\xi) c .
$$


$S(\eta)$ satisfies the master equation

$$
\frac{1}{2}(\mathcal{S}(\eta), \mathcal{S}(\eta))+c \frac{\partial \mathcal{S}(\eta)}{\partial \alpha}=\left(\frac{\partial S(\xi)}{\partial \alpha}-f^{A}(\xi) \frac{\delta S(\xi)}{\delta \varphi^{A}}\right) c=0
$$

where the antibracket $(F, G)$ for functionals $F$ and $G$ is defined as:

$$
(F, G)=F \frac{\overleftarrow{\delta}}{\delta \varphi^{A}} \frac{\delta}{\delta \varphi_{A}^{*}} G-F \frac{\overleftarrow{\delta}}{\delta \varphi_{A}^{*}} \frac{\delta}{\delta \varphi^{A}} G
$$

The only consequence of master equation (5) is eq. (2) for the functional $\left.S(\eta)\right|_{\varphi^{*}=0}=$ $S(\xi)$, whose general solution is

$$
S(\xi)=S_{0}(\Phi(\xi))
$$

with some functional $S_{0}$. So, if $S(\xi)$ has form (1), the master action $\mathcal{S}(\eta)$ satisfies the master equation. Inversely, if we require for the master action $\mathcal{S}(\eta)$ to satisfy master equation (5), then the action $S(\xi)$ will have the form (1).

The Green function generating functional is defined by

$$
\begin{gathered}
Z(J)=e^{\frac{i}{\hbar} W(J)}=\langle 1\rangle, \\
\langle Q\rangle \equiv\left\langle Q(\eta) e^{\frac{i}{\hbar}\left(\mathcal{S}_{i n t}(\eta)+J_{A} \varphi^{A}\right)}\right\rangle_{r e n}, \\
\mathcal{S}_{i n t}(\eta)=\mathcal{S}(\eta)-S_{2}(\varphi)
\end{gathered}
$$

where $S_{2}(\varphi)=\left.\mathcal{S}(\eta)\right|_{g=\varphi^{*}=0}, Q(\eta)$ is an arbitrary functional, and $\langle(\ldots)\rangle_{\text {ren }}$ implies the mean over the free vacuum of the expression in the parenthesis using the Feynman rules with the free propagators defined by action $S_{2}(\varphi)$ and some regularization and renormalization procedure. We do not need an explicit form of the regularization scheme, however we assume that the quantum action principle is valid for the finite Green functions (see [18] and reference therein; all the scheme used at present satisfy this assumption). In particular, the following properties are valid $(\mathcal{T}(\eta)$ is the vertex function generating functional for the theory with action $\mathcal{S}(\eta)$ ):

(i)

$$
\frac{\partial}{\partial \lambda} \mathcal{T}(\eta)=Q(\hbar, \eta) \circ \mathcal{T}(\eta), \quad Q(\hbar, \eta)=\frac{\partial}{\partial \lambda} \mathcal{S}(\eta)+\hbar Q^{(1)}(\hbar, \eta),
$$

where the operation (the so called insertion) $Q(\hbar, \eta) \circ \mathcal{T}(\eta)$ implies that the vertex function generating functional are evaluated in accordance with the standard Feynman rules with the additional vertex $Q(\hbar, \eta), \lambda$ is an arbitrary parameter of the theory under consideration, and $Q^{(1)}(\hbar, \eta)$ is some local functional, which is equal to zero if parameter $\lambda$ does not appear in the free propagators (i.e. it enters only $\mathcal{S}_{\text {int }}$ ).

(ii)

$$
\frac{\delta}{\delta \varphi^{A}} \mathcal{T}(\eta)=Q_{A}(\hbar, \eta) \circ \mathcal{T}(\eta), \quad Q_{A}(\hbar, \eta)=\frac{\delta}{\delta \varphi^{A}} \mathcal{S}(\eta)+\hbar Q_{A}^{(1)}(\hbar, \eta)
$$

where $Q_{A}^{(1)}(\hbar, \eta)$ are some local functionals. 
(iii) The vertex function generating functional $\mathcal{T}(\eta)$ is Poincare invariant and has all the linear homogeneous symmetries of the action functional $\mathcal{S}(\eta)$ that do not touch the space-time coordinates and Lorentzian indices. In particular, in the case under consideration, the vertex function generating functional conserves the ghost number.

The regularization properties (i), (ii) enable us to establish [18] that the vertex function generating functional satisfies eq. (5) up to the local insertions:

$$
\begin{gathered}
\frac{1}{2}(\mathcal{T}(\eta), \mathcal{T}(\eta))+c \frac{\partial \mathcal{T}(\eta)}{\partial \alpha}=-\hbar Q^{(1)}(\hbar, \eta) \circ \mathcal{T}(\eta), \\
Q^{(1)}(\hbar, \eta)=Q_{0}^{(1)}(\eta)+O(\hbar) .
\end{gathered}
$$

The local insertions must satisfy the equation that is the consistency condition for eq. (6):

$$
\left(\mathcal{T}(\eta), Q^{(1)}(\hbar, \eta) \circ \mathcal{T}(\eta)\right)+c \frac{\partial}{\partial \alpha}\left(Q^{(1)}(\hbar, \eta) \circ \mathcal{T}(\eta)\right)=0
$$

We have in the one-loop approximation

$$
\begin{aligned}
& \left(\mathcal{T}_{[1]}(\eta), \mathcal{T}_{[1]}(\eta)\right)_{[1]}=-\hbar Q_{0}^{(1)}(\eta), \\
& \mathcal{T}(\eta)=\mathcal{S}(\eta)+\hbar \mathcal{T}_{1}(\eta)+O\left(\hbar^{2}\right),
\end{aligned}
$$

and the lower index " $[n]$ " at any functional $G$ implies that only the first $n+1$ terms of the Teylor series in $\hbar$ are taken into account:

$$
G \equiv G_{[n]}+O\left(\hbar^{n+1}\right), \quad \frac{\partial^{n+1}}{\partial \hbar^{n+1}} G_{[n]}=0
$$

Because of the ghost number conservation, the functional $Q_{0}^{(1)}(\eta)$ has a ghost number 1 , therefore, it is linear in $c$ and does not depend on $\varphi_{A}^{*}$ :

$$
Q_{0}^{(1)}(\eta)=c q^{(1)}(\xi)
$$

The consistency condition in the one-loop approximation

$$
\begin{gathered}
\omega Q_{0}^{(1)}(\eta)=0 \\
\omega=\frac{\delta \mathcal{S}(\eta)}{\delta \varphi^{A}} \frac{\delta}{\delta \varphi_{A}^{*}}+\frac{\delta \mathcal{S}(\eta)}{\delta \varphi_{A}^{*}} \frac{\delta}{\delta \varphi^{A}}+c \frac{\partial}{\partial \alpha}, \quad \omega^{2}=0
\end{gathered}
$$

is identically satisfied.

Lemma:

The functional $Q_{0}^{(1)}$ can be represented as

$$
Q_{0}^{(1)}(\eta)=\omega X^{(1)}(\eta)
$$

with some local functional $X^{(1)}(\eta), g h\left(X^{(1)}\right)=0$.

To prove the Lemma, it is convenient to pass from variables $\eta$ to variables $\tilde{\eta}=$ $\left\{\Phi^{A}, \Phi_{A}^{*}=\varphi_{B}^{*}\left(\partial \varphi^{B}(\alpha, \Phi) / \partial \Phi^{A}\right), \alpha, c\right\}$. With any functional $G(\eta)$, we also associate the functional $\tilde{G}(\tilde{\eta})$ :

$$
\tilde{G}(\tilde{\eta})=G(\eta(\tilde{\eta})), \quad G(\eta)=\tilde{G}(\tilde{\eta}(\eta))
$$


In this case

$$
\begin{gathered}
\omega G(\eta)=\tilde{\omega} \tilde{G}(\tilde{\eta}), \\
\tilde{\omega}=\frac{\delta S_{0}(\Phi)}{\delta \Phi^{A}} \frac{\delta}{\delta \Phi_{A}^{*}}+c \frac{\partial}{\partial \alpha} .
\end{gathered}
$$

In new variables, the statement of the Lemma considered as on $X^{(1)}(\eta)$ has the form:

$$
\tilde{Q}_{0}^{(1)}(\tilde{\eta})=\tilde{\omega} \tilde{X}^{(1)}(\tilde{\eta}) .
$$

To solve this equation, we introduce the operator $\gamma=\alpha \partial / \partial c$. The operators $\omega$ and $\gamma$ forms an algebra:

$$
\begin{gathered}
\omega^{2}=\gamma^{2}=0, \quad \omega \gamma+\gamma \omega=N, \quad[\omega, N]=[\gamma, N]=0, \\
N=\alpha \frac{\partial}{\partial \alpha}+c \frac{\partial}{\partial c} .
\end{gathered}
$$

A particular solution $\tilde{X}_{p}^{(1)}(\tilde{\eta})$ of (nonhomogeneous) eq. (17) can be taken in the form:

$$
\tilde{X}_{p}^{(1)}(\tilde{\eta})=\frac{1}{N} \gamma \tilde{Q}_{0}^{(1)}(\tilde{\eta}),
$$

where the action of an arbitrary function $f(N)$ of operator $N$ is defined by:

$$
f(N) \alpha^{k} c^{l}=f(k+l) \alpha^{k} c^{l}, \quad k, l \geq 0 .
$$

We note that $\tilde{X}_{p}^{(1)}(\tilde{\eta})$ does not depend on $\varphi^{*}$ and $c: \tilde{X}_{p}^{(1)}=\tilde{X}_{p}^{(1)}(\tilde{\xi})$. The general solution to eq. (7) is obtained by adding the general solution $\tilde{X}_{h}^{(1)}(\tilde{\eta})$ of the homogeneous equation

$$
\tilde{\omega} \tilde{X}_{h}^{(1)}(\tilde{\eta})=0
$$

to $\tilde{X}_{p}^{(1)}(\tilde{\xi})$. We present $\tilde{X}_{h}^{(1)}(\tilde{\eta})$ as

$$
\tilde{X}_{h}^{(1)}(\tilde{\eta})=S_{01}(\Phi)+\tilde{X}_{h 1}^{(1)}(\tilde{\eta}), \quad S_{01}(\Phi)=\left.\tilde{X}_{h}^{(1)}(\tilde{\eta})\right|_{\alpha=c=0}
$$

$\left(S_{01}\right.$ depends only on $\Phi$ because $\left.g h\left(\tilde{X}_{h}^{(1)}\right)=0\right)$. The functional $S_{01}(\Phi)$ do not enter eq. (8) and the standard arguments give:

$$
\tilde{X}_{h 1}^{(1)}(\tilde{\eta})=\tilde{\omega} \tilde{Y}^{(1)}(\tilde{\eta}), \quad g h\left(\tilde{Y}^{(1)}\right)=-1,
$$

with some local functional $\tilde{Y}^{(1)}(\tilde{\eta})=\Phi_{A}^{*} \tilde{\Phi}_{1}^{A}(\tilde{\xi})$.

Returning to the initial variables, we obtain:

$$
\begin{gathered}
X^{(1)}(\eta)= \\
=S_{01}(\Phi)+\frac{\delta S_{0}(\Phi)}{\delta \Phi^{A}} \Phi_{1}^{A}(\xi)-\varphi_{A}^{*} \frac{\partial \tilde{\varphi}^{A}}{\partial \Phi_{B}}\left(\frac{\partial \Phi_{1}^{B}(\xi)}{\partial \alpha}-\frac{\partial \Phi_{1}^{B}(\xi)}{\partial \varphi^{C}} \frac{\partial \tilde{\varphi}^{C}}{\partial \Phi^{D}} \frac{\partial \Phi^{D}(\xi)}{\partial \alpha}\right) c+X_{p}^{(1)}(\xi) .
\end{gathered}
$$

We consider now the master action $\mathcal{S}^{(1)}(\eta)$ :

$$
\mathcal{S}^{(1)}(\eta)=\mathcal{S}(\eta)+\hbar X^{(1)}(\eta) \equiv S^{(1)}(\xi)-\varphi_{A}^{*} f^{(1) A}(\xi) c
$$


the function $f^{(1) A}(\xi)=f^{A}(\xi)+O(\hbar)$ being local. The sense of the separate terms in expression (9) for $X^{(1)}$ becomes now clear: the first term describes the quantum corrections to the initial classical action, the second and third ones describe the quantum corrections to the change of variables, the last term has to "compensate" a possible noncovariance of the regularization scheme adopted.

The master action $\mathcal{S}^{(1)}(\eta)$ does not satisfy master equation (5):

$$
\left(\mathcal{S}^{(1)}(\eta), \mathcal{S}^{(1)}(\eta)\right)+c \frac{\partial \mathcal{S}^{(1)}(\eta)}{\partial \alpha}=\hbar \Lambda^{(1)}(\hbar, \eta)
$$

It is however important, that $\Lambda^{(1)}(\hbar, \eta)$ is a local functional.

The vertex function generating functional $\mathcal{T}^{(1)}(\eta)$ in the theory with the action $\mathcal{S}^{(1)}(\eta)$ does satisfy the master equation up to local insertion. But it is easy to verify that the local insertions are absent in the one-loop approximation:

$$
\begin{gathered}
\mathcal{T}_{[1]}^{(1)}(\eta)=\mathcal{T}_{[1]}(\eta)+\hbar X^{(1)}(\eta) \\
\frac{1}{2}\left(\mathcal{T}_{[1]}^{(1)}(\eta), \mathcal{T}_{[1]}^{(1)}(\eta)\right)_{[1]}+c \frac{\partial}{\partial \alpha} \mathcal{T}_{[1]}(\eta)=\frac{1}{2}\left(\mathcal{T}_{[1]}(\eta), \mathcal{T}_{[1]}(\eta)\right)_{[1]}+c \frac{\partial}{\partial \alpha} \mathcal{T}_{[1]}(\eta)+ \\
+\left(\mathcal{T}^{(1)}(\eta)_{[1]}, \hbar X^{(1)}(\eta)\right)_{[1]}+\hbar c \frac{\partial}{\partial \alpha} X^{(1)}(\eta)=-\hbar Q_{0}^{(1)}(\eta)+\hbar \omega X^{(1)}(\eta)=0 .
\end{gathered}
$$

So, the violation of the master equation for $\mathcal{T}^{(1)}(\eta)$ begins with the two-loop approximation:

$$
\begin{gathered}
\frac{1}{2}\left(\mathcal{T}^{(1)}(\eta), \mathcal{T}^{(1)}(\eta)\right)+c \frac{\partial}{\partial \alpha} \mathcal{T}^{(1)}(\eta)=-\hbar^{2} Q^{(2)}(\hbar, \eta) \circ\left(\mathcal{T}^{(1)}(\eta),\right. \\
\left(\mathcal{T}^{(1)}(\eta), Q^{(2)}(\hbar, \eta) \circ\left(\mathcal{T}^{(1)}(\eta)\right)+c \frac{\partial}{\partial \alpha}\left(Q^{(2)}(\hbar, \eta) \circ\left(\mathcal{T}^{(1)}(\eta)\right)=0 .\right.\right.
\end{gathered}
$$

By induction, we finally obtain: There exists the action $\mathcal{S}^{(\infty)}(\eta)$,

$$
\mathcal{S}^{(\infty)}(\eta)=\mathcal{S}(\eta)+\sum_{n=1} \hbar^{n} X^{(n)}(\eta) \equiv S^{(\infty)}(\xi)-\varphi_{A}^{*} f^{(\infty) A}(\xi) c
$$

where $f^{(\infty) A}(\xi)$ are local functions, such that the vertex function generating functional $\mathcal{T}^{(\infty)}(\eta)$ satisfies the master equation:

$$
\frac{1}{2}\left(\mathcal{T}^{(\infty)}(\eta), \mathcal{T}^{(\infty)}(\eta)\right)+c \frac{\partial}{\partial \alpha} \mathcal{T}^{(\infty)}(\eta)=0
$$

With the account of the relation (that holds in view of property (i) of the regularizations used)

$$
\frac{\delta}{\delta \varphi_{A}^{*}} \mathcal{T}^{(\infty)}(\eta)=-c\left\langle f^{(\infty) A}\right\rangle^{(\infty)}(\xi),
$$

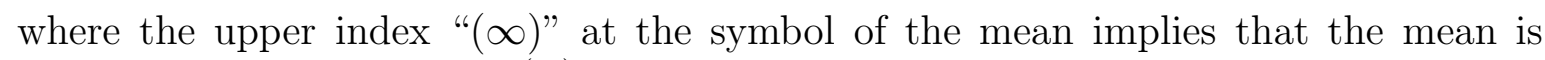
calculated with the action $S^{(\infty)}(\xi)$, we obtain:

$$
\frac{\partial}{\partial \alpha} \Gamma^{(\infty)}(\xi)-\left\langle f^{(\infty) A}\right\rangle^{(\infty)}(\xi) \frac{\delta}{\delta \varphi^{A}} \Gamma^{(\infty)}(\xi)=0,
$$


where $\left.\Gamma^{(\infty)}(\xi) \equiv \mathcal{T}(\eta)\right|_{\varphi *=0}$ is the vertex function generating functional for the theory with the "renormalized" action $S^{(\infty)}(\xi)$.

Thus, it is established that by adding the appropriate counterterms to the initial action we can always make the vertex function generating functional to satisfy basic equation (4), i.e. the equivalence theorem to be fulfilled.

\section{The example}

In this section, we are consider the example of the family of classical theories related by the change of variables whose quantization leads to the physically nonequivalent theories, the equivalence theorem being valid.

The model is described by action

$$
\begin{gathered}
S(\alpha, \psi)=S_{0}(\Psi(\alpha, \psi))=\bar{\psi}\left(i \gamma^{\mu} \partial_{\mu}+\gamma^{\mu} V_{\mu}+\gamma^{\mu} \gamma^{5} A_{\mu}+\alpha \frac{f_{\pi}}{m} \gamma^{\mu} \gamma^{5} \partial_{\mu} \varphi\right) \psi, \\
S_{0}(\psi)=\bar{\psi}\left(i \gamma^{\mu} \partial_{\mu}+\gamma^{\mu} V_{\mu}+\gamma^{\mu} \gamma^{5} A_{\mu}\right) \psi, \quad \Psi(\alpha, \psi)=e^{-i \alpha \frac{f_{\pi}}{m} \varphi} \psi,
\end{gathered}
$$

where $\psi(x)$ is a quantum Dirac field, $V_{\mu}(x), A_{\mu}(x), \varphi(x)$ are respectively external vector, axial and pseudoscalar fields, $\gamma^{5}=i \gamma^{0} \gamma^{1} \gamma^{2} \gamma^{3}$, the metrics is $\operatorname{diag}(+,-,-,-)$, the symbol $\int d x$ is omitted.

The vertex function generating functional must satisfy the equation:

$$
\frac{\partial}{\partial \alpha} \Gamma^{(1)}-\left\langle f_{\psi}^{(1)}\right\rangle^{(1)} \frac{\delta}{\delta \psi} \Gamma^{(1)}-\left\langle f_{\bar{\psi}}^{(1)}\right\rangle^{(1)} \frac{\delta}{\delta \bar{\psi}} \Gamma^{(1)}=0
$$

where the upper index "(1)" means that the theory is exhausted by the one-loop approximation, $f_{\psi}^{(1)}=\left(-i \frac{f_{\pi}}{m} \varphi \gamma^{5}+O(\hbar)\right) \psi, f_{\bar{\psi}}^{(1)}=\bar{\psi}\left(-i \frac{f_{\pi}}{m} \varphi \gamma^{5}+O(\hbar)\right)$,

We restrict ourselves to the discussion of vacuum diagrams, i.e. of the vertex function generating functional for zero arguments $\psi \bar{\psi}$ :

$$
\left.\bar{\Gamma} \equiv \Gamma\right|_{\psi=\bar{\psi}=0}=\bar{\Gamma}\left(\alpha, V_{\mu}, A_{\mu}, \varphi\right)
$$

In this limit $f_{\psi}^{(1)}=f_{\bar{\psi}}^{(1)}=0$, so that $\bar{\Gamma}$ must satisfy the equation

$$
\frac{\partial}{\partial \alpha} \bar{\Gamma}=0
$$

For $\varphi=0$ the expression $\tilde{\Gamma}\left(V_{\mu}, A_{\mu}\right)=\left.\bar{\Gamma}\right|_{\varphi=0}$ is uniquely defined by the requirement of the exact conservation of the vector current:

$$
\partial_{\mu} \frac{\delta}{\delta V_{\mu}(x)} \tilde{\Gamma}=0
$$

and the conservation of the axial current, excepting the diagrams with three external lines. In this case

$$
\partial_{\mu} \frac{\delta}{\delta A_{\mu}(x)} \tilde{\Gamma}=-\frac{\hbar}{4 \pi^{2}}\left(\varepsilon^{\mu \nu \lambda \sigma} \partial_{\mu} V_{\nu}(x) \partial_{\lambda} V_{\sigma}(x)+\frac{1}{3} \varepsilon^{\mu \nu \lambda \sigma} \partial_{\mu} A_{\nu}(x) \partial_{\lambda} A_{\sigma}(x)\right),
$$


$\varepsilon^{0123}=1$. For $\varphi \neq 0$ the expression for $\bar{\Gamma}$ is derived from the expression for $\tilde{\Gamma}$ by the substitution of $A_{\mu}+\alpha \frac{f_{\pi}}{m} \partial_{\mu} \varphi$ for $A_{\mu}$ and by the addition of possible local counterterms (the conservation of the vector current is required still):

$$
\bar{\Gamma}\left(\alpha, V_{\mu}, A_{\mu}, \varphi\right)=\tilde{\Gamma}\left(V_{\mu}, A_{\mu}+\alpha \frac{f_{\pi}}{m} \partial_{\mu} \varphi\right)+\hbar S_{\text {contr }}\left(\alpha, V_{\mu}, A_{\mu}, \varphi\right) .
$$

The dependence of $\tilde{\Gamma}$ on $\varphi$ may be calculated explicitly (for example, through a differentiation by $\alpha$ ):

$$
\begin{gathered}
\tilde{\Gamma}\left(V_{\mu}, A_{\mu}+\alpha \frac{f_{\pi}}{m} \partial_{\mu} \varphi\right)= \\
=\tilde{\Gamma}\left(V_{\mu}, A_{\mu}\right)+\frac{\alpha \hbar}{4 \pi^{2}} \frac{f_{\pi}}{m} \int d x\left(\varphi(x) \varepsilon^{\mu \nu \lambda \sigma} \partial_{\mu} V_{\nu}(x) \partial_{\lambda} V_{\sigma}(x)+\frac{1}{3} \varphi(x) \varepsilon^{\mu \nu \lambda \sigma} \partial_{\mu} A_{\nu}(x) \partial_{\lambda} A_{\sigma}(x)\right) .
\end{gathered}
$$

As for $S_{\text {contr }}\left(\alpha, V_{\mu}, A_{\mu}, \varphi\right)$, we shall only extract the term linear in $\varphi$ and containing the tensor $\varepsilon^{\mu \nu \lambda \sigma}$. The terms having other independent structure linear in $\varphi$ as well as the terms of the qudaratic and higher powers in $\varphi$ are inessential for us:

$$
\begin{gathered}
S_{\text {contr }}\left(\alpha, V_{\mu}, A_{\mu}, \varphi\right)= \\
=\frac{f_{\pi}}{m} \varphi\left(r_{1}^{\prime}(\alpha) \varepsilon^{\mu \nu \lambda \sigma} \partial_{\mu} V_{\nu} \partial_{\lambda} V_{\sigma}+r_{2}^{\prime}(\alpha) \varepsilon^{\mu \nu \lambda \sigma} \partial_{\mu} A_{\nu} \partial_{\lambda} A_{\sigma}\right)+S_{c o n t r}^{\prime}\left(\alpha, V_{\mu}, A_{\mu}, \varphi\right),
\end{gathered}
$$

where the sign of $\int d x$ is omitted. Thus the general expression of $\bar{\Gamma}$ reads:

$$
\begin{gathered}
\bar{\Gamma}\left(\alpha, V_{\mu}, A_{\mu}, \varphi\right)=\tilde{\Gamma}\left(V_{\mu}, A_{\mu}\right)+ \\
+\hbar \frac{f_{\pi}}{m} \varphi\left(r_{1}(\alpha) \varepsilon^{\mu \nu \lambda \sigma} \partial_{\mu} V_{\nu} \partial_{\lambda} V_{\sigma}+r_{2}(\alpha) \varepsilon^{\mu \nu \lambda \sigma} \partial_{\mu} A_{\nu} \partial_{\lambda} A_{\sigma}\right)+\hbar S_{c o n t r}^{\prime}\left(\alpha, V_{\mu}, A_{\mu}, \varphi\right), \\
r_{1}(\alpha)=\frac{\alpha}{4 \pi^{2}}+r_{1}^{\prime}(\alpha), \quad r_{2}(\alpha)=\frac{\alpha}{12 \pi^{2}}+r_{2}^{\prime}(\alpha) .
\end{gathered}
$$

The equation (11) is satisfied for the following choice of counterterms:

$$
\begin{gathered}
r_{1}(\alpha)=-\frac{\alpha}{4 \pi^{2}}+r_{1}, \quad r_{2}(\alpha)=-\frac{\alpha}{12 \pi^{2}}+r_{2}, \quad r_{1}, r_{2}=\text { const } \\
S_{\text {contr }}^{\prime}=S_{\text {contr }}^{\prime}\left(V_{\mu}, A_{\mu}, \varphi\right),
\end{gathered}
$$

$\left(r_{1}, r_{2} S_{\text {contr }}^{\prime} \alpha\right)$

As a result, we get the following expression for $\bar{\Gamma}$ :

$$
\begin{gathered}
\bar{\Gamma}\left(\alpha, V_{\mu}, A_{\mu}, \varphi\right)=\tilde{\Gamma}\left(V_{\mu}, A_{\mu}\right)+ \\
+\hbar \frac{f_{\pi}}{m} \varphi\left(r_{1} \varepsilon^{\mu \nu \lambda \sigma} \partial_{\mu} V_{\nu} \partial_{\lambda} V_{\sigma}+r_{2} \varepsilon^{\mu \nu \lambda \sigma} \partial_{\mu} A_{\nu} \partial_{\lambda} A_{\sigma}\right)+\hbar S_{\text {contr }}^{\prime}\left(V_{\mu}, A_{\mu}, \varphi\right) .
\end{gathered}
$$

This expression clearly satisfies the equivalence theorem (it does not depend on the change of variables in the classical action), however an ambiguity in the choice of counterterms still remains. This ambiguity could be explored in different ways.

If one starts from a quantum theory which is constructed from the classical action (10) $\alpha=0$ :

$$
\left.S(0, \psi)=S_{0}(\psi)\right)=\bar{\psi}\left(i \gamma^{\mu} \partial_{\mu}+\gamma^{\mu} V_{\mu}+\gamma^{\mu} \gamma^{5} A_{\mu}\right) \psi
$$


then it seems natural to require that for $\alpha=0$, and, consequently for any $\alpha$ on the fermion mass shell the quantum theory does not depend on the field $\varphi$. This means that the functional $\bar{\Gamma}$ does not depend on $\varphi$ :

$$
\bar{\Gamma}=\tilde{\Gamma}\left(V_{\mu}, A_{\mu}\right)
$$

On the other hand, if one starts from the quantum theory which is constructed from the classical action (10) for $\alpha=1$ (the choice of any other $\alpha \neq 0$ as a normalization point reduces to a redefinition of the parameter $f_{\pi}$ or $m$ ):

$$
S(1, \psi)=\bar{\psi}\left(i \gamma^{\mu} \partial_{\mu}+\gamma^{\mu} V_{\mu}+\gamma^{\mu} \gamma^{5} A_{\mu}+\frac{f_{\pi}}{m} \gamma^{\mu} \gamma^{5} \partial_{\mu} \varphi\right) \psi
$$

then it seems natural to demand that the field $\frac{f_{\pi}}{m} \partial_{\mu} \varphi$ and the axial field $A_{\nu}$ should interact with the same axial current. In this case we must choose $r_{1}=1 / 4 \pi^{2}, r_{2}=$ $1 / 12 \pi^{2}$ (in addition, we put $S_{\text {contr }}^{\prime}=0$ for simplicity):

$$
\bar{\Gamma}=\tilde{\Gamma}\left(V_{\mu}, A_{\mu}\right)+\frac{\hbar}{4 \pi^{2}} \frac{f_{\pi}}{m} \varphi\left(\varepsilon^{\mu \nu \lambda \sigma} \partial_{\mu} V_{\nu} \partial_{\lambda} V_{\sigma}+\frac{1}{3} \varepsilon^{\mu \nu \lambda \sigma} \partial_{\mu} A_{\nu} \partial_{\lambda} A_{\sigma}\right) .
$$

Thus the example considered demonstrates that the requirement of validity of the equivalence theorem does not eliminate the ambiguity related to possible addition of finite counterterms.

Acknowledgments. The work is supported by Russian Foundation for Basic Researches and by Human Capital and Mobility Program of the European Community, grants RFBR-99-01-00980, RFBR-99-02-17916, INTAS-96-0308.

\section{References}

[1] Dyson F.J., Phys. Rev., 1948, 73, 829.

[2] Borchers H.I., Nuovo Cim., 1960, 15, 784.

[3] Chisholm S.R., Nucl. Phys., 1961, 26, 469.

[4] Kamefuchi S., O’Raifeartaigh L., Salam A., Nucl. Phys., 1961, 28, 529.

[5] Coleman S., Wess I., Zumino B., Phys. Rev., 1968, 177, 2239.

[6] 't Hooft G., Veltman M., "Combinatorics of gauge fields", preprint Utrecht, 1972.

[7] Kallosh R.E., Tyutin I.V., Yadern. Phys., 1973, 17, 190.

[8] Lam Y.M.P., Phys. Rev., 1973, D7, 2943.

[9] Bergere M.C., Lam Yuk-Ming P., Phys. Rev., 1976, D13, 3247.

[10] Tyutin I.V., Yadern. Phys., 1982, 35, 222. 
[11] Bogoliubov N.N., Logunov A.A., Oksak A.I., Todorov I.T., General principles of quantum field theory, Dordrecht-Boston-London, Kluwer, 1990.

[12] Bogoliubov N.N., Shirkov D.V., Introduction to the Theory of Quantum Fields [in Russian], Nauka, Moskow (1976); English transl. prev. ed., Wiley, New York (1959).

[13] Zavialov O I., Renormalized quantum field theory, Dordrecnt, Kluwer, 1990.

[14] Batalin I.A., Vilkovisky G.A., Phys. Lett., 1981, B102, 27.

[15] Voronov B.L., Tyutin I.V., Yadern. Phys., 1981, 33, 1137.

[16] Becchi C., Blasi A., Bonneau G., Collina R., Delduc F., Commun. Math. Phys., 1988, 120, 121.

[17] Brandt Friedemann, Henneaux Marc, Wilch André, Nucl. Phys., 1998, B510, 640.

[18] Piguet O., Sorella S.P., “Algebraic Renormalization”, Springer - Verlag, 1995.

[19] Barnich Glenn, Brandt Friedman, Henneaux Marc, Commun. Math. Phys., 1995, $174,57$. 